\title{
Non-Intrusive Occupancy Monitoring using Smart Meters
}

\author{
Dong Chen, Sean Barker, Adarsh Subbaswamy ${ }^{\dagger}$, David Irwin, and Prashant Shenoy \\ University of Massachusetts Amherst \\ Vanderbilt University ${ }^{\dagger}$
}

\begin{abstract}
Detailed information about a home's occupancy is necessary to implement many advanced energy-efficiency optimizations. However, monitoring occupancy directly is intrusive, typically requiring the deployment of multiple environmental sensors, e.g., motion, acoustic, $\mathrm{CO}_{2}$, etc. In this paper, we explore the potential for Non-Intrusive Occupancy Monitoring (NIOM) by using electricity data from smart meters to infer occupancy. We first observe that a home's pattern of electricity usage generally changes when occupants are present due to their interact with electrical loads. We empirically evaluate these interactions by monitoring ground truth occupancy in two homes, then correlating it with changes in statistical metrics of smart meter data, such as power's mean and variance, over short intervals. In particular, we use each metric's maximum value at night as a proxy for its maximum value in an unoccupied home, and then signal occupancy whenever the daytime value exceeds it. Our results highlight NIOM's potential and its challenges.
\end{abstract}

\section{Categories and Subject Descriptors}

J.7 [Computer Applications]: Computers in Other SystemsCommand and control

\section{General Terms}

Design, Measurement, Management

\section{Keywords}

Energy, Electricity, Grid

\section{INTRODUCTION}

Based on recent estimates, commercial and residential buildings continue to account for $75 \%$ of the electricity usage in the United States [23], with residential buildings alone accounting for nearly $45 \%$. ${ }^{1}$ As a result, improving building energy-efficiency is becoming an increasingly important research area. One of the simplest

\footnotetext{
${ }^{1}$ Research supported by NSF grants CNS-1253063, CNS-1143655, CNS-0916577, CNS-0855128, CNS-0834243, CNS-0845349.

Permission to make digital or hard copies of all or part of this work for personal or classroom use is granted without fee provided that copies are not made or distributed for profit or commercial advantage and that copies bear this notice and the full citation on the first page. Copyrights for components of this work owned by others than ACM must be honored. Abstracting with credit is permitted. To copy otherwise, or republish, to post on servers or to redistribute to lists, requires prior specific permission and/or a fee. Request permissions from Permissions@acm.org.

BuildSys'13, November 14 - 15, 2013, Roma, Italy.

Copyright 2013 ACM 978-1-4503-2431-1/13/11 ...\$15.00.

http://dx.doi.org/10.1145/2528282.2528294.
}

ways to reduce wasted energy and improve energy-efficiency is to automatically regulate a building's electricity usage in real time based on its occupancy $[2,15,19,25]$. Researchers have proposed many applications that use occupancy information to drive a variety of novel building management optimizations. For example, a building management system may save energy in unoccupied buildings by automatically i) disconnecting inactive electrical devices (or loads), such as televisions, gaming consoles, and cable boxes, to lower vampire power usage [11], ii) adjusting environmental setpoints and guardbands for air conditioners, heaters, and humidifiers to operate outside occupants' normal comfort level, thereby shortening the length of their duty cycle [2, 14, 24], iii) turning off or dimming lights aggressively to reduce energy waste [9], and iv) placing idle desktop computers and other IT equipment into lowpower standby states, e.g., Suspend-to-RAM [3].

A key prerequisite for implementing these occupancy-driven applications is an accurate, inexpensive, and non-intrusive method for monitoring occupancy. While determining occupancy is possible using GPS information from smartphones, the approach requires active participation by all occupants: they must carry their phone at all times and integrate it with each building's occupancy-detection system. The approach is clearly not feasible if occupants do not own smartphones, as with many young and low-income users, or are unwilling to link their phones with building management systems due to privacy concerns. Thus, many systems monitor occupancy by strategically deploying one or more environmental sensors, including motion $[2,24,26]$, door [1], acoustic [8, 17], camera $[14,15,20]$, contact $[26,29]$, and $\mathrm{CO} 2[10,31]$ sensors.

As this prior research shows, directly sensing occupancy using environmental sensors poses many challenges. For example, users must carefully place and calibrate motion sensors to prevent spurious detection from pets or events outside windows [24]. While $\mathrm{CO}_{2}$ sensors are less sensitive to placement position and external events, users must precisely calibrate them based on a building's ventilation system. Furthermore, these sensors incur an inherent detection delay, as additional occupants cause $\mathrm{CO}_{2}$ levels to increase slowly [14, 31]. Each of the other environmental sensing options above introduce their own similar types of domain-specific challenges. In general, such direct occupancy monitoring also requires purchasing and retrofitting buildings with many external sensors. As a result, these systems must address problems common to any large distributed system, including detecting, diagnosing, and correcting sensor failures and ensuring each sensor has a reliable network connection. Finally, since retrofitted sensors are typically not connected to the power grid, they require periodic battery replacement or recharging, which imposes a significant maintenance burden in distributed deployments that employ many sensors [18].

The limitations above have led researchers to study indirectly 


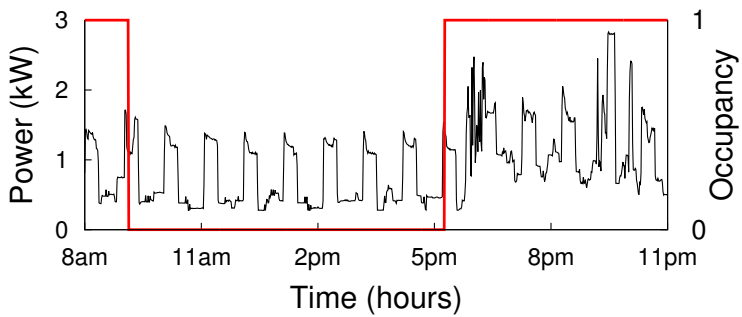

(a) Home-A

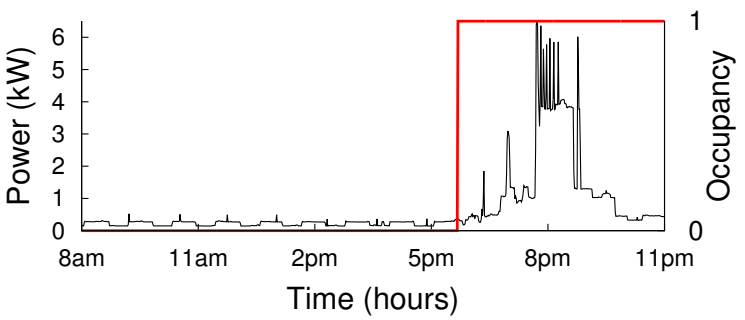

(b) Home-B

Figure 1: Overlay of average power usage every minute (black) with binary occupancy (red), where one indicates at least one occupant is present and zero indicates no occupants are present, over one day (8am-11pm) for two homes.

monitoring occupancy using contextual information sources, including wired [7, 28] and wireless network traffic [4, 16, 25] and online sources [30], such as calendars and chat applications. Indirect approaches correlate one or more activities with occupancy, and are cheaper and less intrusive than direct approaches, as they do not require deploying and maintaining extra sensors. However, accuracy depends on occupants regularly performing specific activities, e.g., generating network traffic or updating a calendar.

In this paper, we empirically explore the potential of indirectly monitoring occupancy solely through electricity use-possibly the most common occupant activity. In particular, we focus on monitoring occupancy in residential homes using the coarse-grained data produced by commodity smart meters, which record a home's electricity usage anywhere between every one to fifteen minutes. Typical smart meter data clearly demonstrates that a home's pattern of electricity usage changes whenever occupants are present. Figure 1 illustrates this point by overlaying average power usage every minute with binary occupancy (one is occupied, zero is unoccupied) between $8 \mathrm{am}$ and $11 \mathrm{pm}$ for two homes. Though the homes have different occupants, loads, and daily routines, the pattern of power usage clearly change whenever people are home.

Prior research that detects occupancy from electrical events requires expensive, specialized, and highly-calibrated equipment to record and analyze high-frequency data, e.g., $\gg 2 \mathrm{kHz}$ [27]. In contrast, smart meters are already widely deployed: by 2011, an estimated 493 utilities had collectively installed more than 37 million in the United States [13]. Thus, we target data that is already available to utilities, and does not require deploying any additional sensors. While existing work has demonstrated a correlation between occupancy and coarse-grained electricity usage [26] by using occupancy to infer electricity usage, our work examines the reverse relationship by using electricity usage to infer occupancy. Our hypothesis is that occupants' interaction with electrical loads, e.g., turning them on and off, enables simple Non-Intrusive Occupancy Monitoring (NIOM) by detecting changes in statistical metrics in a home's power data, such as its mean, variance, and range. In evaluating our hypothesis, we make the following contributions:

Data Collection and Analysis. In $\S 2$, we analyze data from two deeply instrumented homes to quantify the opportunity for NIOM. We collect ground truth data on both occupants' physical interactions with the electrical system that imply occupancy, e.g., flipping a switch, and the homes' real occupancy using GPS sensors, and then compare it with the homes' smart meter data. Our analysis not only reveals how the homes' pattern of electricity usage changes when occupants are present, but also why it changes and which types of loads contribute the most occupancy-implying events.

NIOM Algorithm. In \$3, we use the observations from our data analysis to develop a simple NIOM algorithm that monitors occupancy by first detecting occupancy events based on changes in the statistical metrics of power data, and then clustering nearby events together to produce a continuous trace of occupancy. We propose automatically setting metric thresholds by using their value at night as a rough approximation of its value in an unoccupied home.

Implementation and Evaluation. In $\S 4$, we evaluate NIOM's accuracy by comparing with both ground truth occupancy data and an approach based on Non-Intrusive Load Monitoring (NILM) [22], which first extracts the power usage of each load and then detects occupancy from the power usage of interactive loads. Our results show that our simple approach is more effective at separating occupied from unoccupied periods than the NILM-based approach.

Finally, $\$ 5$ concludes by discussing the i) new applications NIOM enables, ii) potential to extend our approach to monitor the number of occupants in a home and their location, and iii) possibility of other simple, useful, and tractable non-intrusive analytics.

\section{DATA COLLECTION AND ANALYSIS}

We instrument two homes (Home-A and Home-B in Figure 1 from the Smart* dataset [6]) with a variety of sensors to collect ground truth data about each home's electrical events and its occupancy. Detailed information about the physical characteristics of both homes is available in recent work [6]. Ground truth data is critical in assessing and evaluating NIOM. We first briefly describe the sensors, the raw data they generate, and how we post-process this data to obtain ground truth data for i) the occupants' physical interactions with electrical loads, e.g., flipping switches and pushing buttons, and ii) the homes' occupancy information. We then compare this data with the homes' smart meter data, and make a series of observations to motivate NIOM. Note that the NIOM algorithm presented in Section 3 only uses a home's smart meter data to infer occupancy. We only use our external sensors to quantify the potential for NIOM in a home.

\subsection{Instrumentation}

Physical Interactions. To monitor occupants' physical interactions with loads, we use multiple eGauge energy monitors [12] installed in the homes' electrical panels to record total (real) grid power usage and the (real) power usage for each of the individual branch circuits every second. We use the branch circuit data to extract events - large changes in power-that imply occupants' physical interactions with loads. Reliably extracting these events directly from the homes' aggregate electricity data is challenging due to the presence of background loads, such as heaters, air conditioners, and refrigerators, that are driven by automated controllers and consequently do not imply occupancy. Notably, automatically extracting these events from a home's aggregate power data is closely related to performing NILM. Since background loads are wired to dedicated circuits in both homes, monitoring branch circuits naturally separates their electricity use from that of interactive loads, 


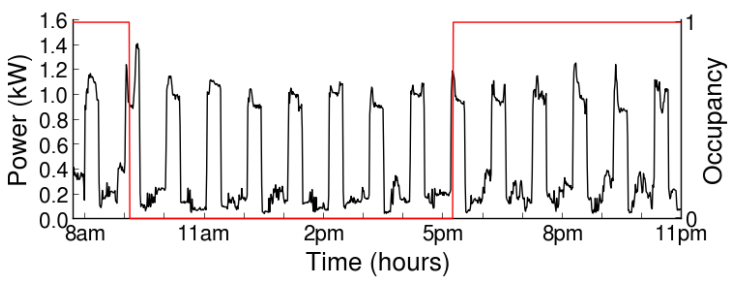

(a) Background Loads

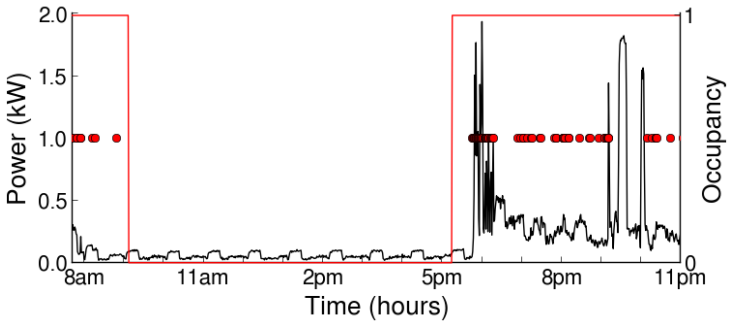

(b) Interactive Loads with Event Labels

Figure 2: Power usage of background (a) and interactive (b) loads from Home-A for the same day as in Figure 1(a). We include event labels in (b) that indicate occupants' physical interactions with electrical loads.

which people manually control (e.g., by turning them on and off). We supplement our raw electricity data by using Insteon-enabled i) wall switches, ii) door sensors, and iii) thermostats. These sensors signal a computer whenever someone physically presses a wall switch, opens a door that triggers an electrical event, such as a freezer door that turns on an interior light, or adjusts a thermostat.

To asses NIOM's potential, we post-process this raw electricity and switch data to automatically generate an event trace of occupants' physical interactions with electrical loads. Examples of these events include occupants using a remote control to turn on a television, pressing a button to cook food in the microwave, or toggling a wall switch. Ultimately, the correlation between a home's pattern of electricity usage and occupancy derives from these physical interactions with electrical loads. Our goal is to learn the types of physical interactions occupants have with electrical loads, as well as their rate and frequency, to quantify the potential to monitor occupancy via smart meter data. For instance, if occupants are home and not interacting with any electrical loads, such as when reading a book, the correlation between electricity usage and occupancy may be weak. To extract events from our electricity data, we focus on circuits that power interactive loads and ignore those dedicated to background loads. Since each of these circuits only supports a small number of loads, we manually profile every load per circuit and derive power event detection rules tailored for each of a circuit's loads. In general, we find that a change in power greater than $30 \mathrm{~W}$ indicates occupant interaction. We then augment these events with any events generated directly from the Insteon wall switch, door, and thermostat sensors to yield a final event trace. Ground Truth Occupancy. In addition to automatically tracking occupants' interaction with electrical loads, we monitor ground truth occupancy via occupants' smartphones. This type of monitoring is feasible because the two primary occupants in each home reliably carry their phones at all times, and the occupants without smartphones are never home without the primary occupants also being home. We gather occupancy data by installing the Google Latitude mobile app on each phone. Latitude enables authenticated users to query a URL to retrieve a phone's current GPS coordinates (latitude and longitude), which we collect from a central server every 30 seconds and translate into a binary occupancy trace by considering locations within 500 meters of the home as 'occupied.' Note that we only use the GPS data for deriving ground truth occupancy, and not when detecting occupancy from smart meter data.

\subsection{Analysis and Observations}

Figure 1 (from §1) illustrates how a home's pattern of electricity usage changes whenever occupants are present. In both homes, the load profile reveals a number of attributes in the data that appear to correlate with occupancy, including a higher mean power usage, a higher minimum power usage, i.e., a raised power floor, a higher maximum power usage, and more bursty power usage. On this day, Home-B exhibits a drastic change in the pattern of power usage when occupants are present. Interestingly, for Home-A, which exhibits the weaker correlation between electricity usage and occupancy, there appear to be periods when occupants are present, but the load profile is similar to the profile when occupants are not present. For example, in Figure 1(a), the home is occupied between $8 \mathrm{am}$ and 9am, but the load profile at this time appears to be similar to the profile from $10 \mathrm{am}$ to $11 \mathrm{am}$, when the home is unoccupied. As we show in $\S 4$, since occupants may not continuously interact with the electrical loads, any system that uses smart meter data to monitor occupancy has the potential to generate false negatives, i.e., by incorrectly determining that no one is home.

We use our event traces to examine the extent of occupancy's correlation with electricity usage, including the frequency of occupants' physical interactions with loads and its impact on home electricity usage. Here, we focus on Home-A, since it exhibits the weaker correlation. For Home-A, in Figure 2, we separate the electricity usage of the interactive loads and background loads for the same day as in Figure 1(a). Figure 2(a) shows the power usage of just the background loads, while Figure 2(b) shows the power usage of just the interactive loads, as well as our event labels that signify occupants' physical interactions. Since users do not interact with background loads, Figure 2(a) has no event labels.

Figure 2 illustrate multiple points about the potential for NIOM. First, note that the background loads in Figure 2(a) have a significant and highly variable load profile between $40 \mathrm{~W}$ and $1.5 \mathrm{~kW}$. In total, on this day, the background loads contribute the majority $(56 \%)$ of the home's electricity usage, while exhibiting a range in power usage of $1366 \mathrm{~W}$, e.g., a peak usage of $1413 \mathrm{~W}$ and a minimum usage of $47 \mathrm{~W}$. As a result, we cannot simply assume that an unoccupied home has a low or constant power usage. However, the background loads do exhibit a consistent pattern of power usage regardless of occupancy. Second, Figure 2(b) indicates that occupants have near-continuous interaction with electrical loads while home, physically interacting with them at an average rate of 11.58 interactions per hour. In total, these interactive loads contribute $44 \%$ of the home's electricity usage, while exhibiting an even wider range of power usage than the background loads, e.g., with a peak usage of $1937 \mathrm{~W}$ and a minimum usage of $21 \mathrm{~W}$. In addition, the longest observed "dead period"—-when occupants are home but do not interact with electrical loads - is only 58 minutes (between 9pm and $10 \mathrm{pm}$ when they are watching TV). Unlike the background loads, the interactive loads only consume significant power when occupants are home. Since aggregate power is the combination of the interactive and background loads, we expect any change in the pattern of power usage to also indicate a change in occupancy.

While we only use one day's worth of data to illustrate the points above, Table 1 shows the number of events over 71 days starting 


\begin{tabular}{|l|c|r|}
\hline Category & Count & Percentage \\
\hline Lights & 3048 & 39.25 \\
\hline Kitchen & 2882 & 37.12 \\
\hline IT & 1374 & 17.69 \\
\hline Large Appliances & 263 & 3.39 \\
\hline Miscellaneous & 198 & 2.55 \\
\hline \hline Total & $\mathbf{7 7 6 5}$ & $\mathbf{1 0 0}$ \\
\hline
\end{tabular}

Table 1: Number of physical interactions with different types of electrical loads over a 71 day period.

in mid-April caused by different types of loads, including lighting, IT devices, e.g., computer, television, and gaming console), kitchen appliances, (e.g., toaster, microwave, and coffee maker), large appliances, (e.g., washing machine and dryer), and miscellaneous (e.g., vacuums and fans). In total, the occupants interacted with loads 7765 over this period at a rate of 109 events per day, and 10.08 events per hour when occupied. Not surprisingly, a large majority of events are due to lighting. As we discuss in $\$ 4$, lighting is a particularly challenging load for NILM algorithms to detect.

While the observations above use one-minute average power data, they also hold for coarser data, although the correlations become more subtle as the data becomes coarser. For example, an occupied home may have $2 \mathrm{X}$ higher average power than an unoccupied home using average power data over five minutes, but only 1.1X higher average power using average power data over one hour. In general, as data becomes coarser, it averages out the "peaky" features of interactive loads in power data that imply occupancy.

\section{NIOM ALGORITHM}

Unlike the analysis in the previous section, where we use external sensors to establish ground truth and study the potential for NIOM, our NIOM algorithm uses only a home's smart meter data to infer occupancy. Below, we describe three statistical metrics we monitor in smart meter data to detect occupancy and our intuition for using them. We then propose a simple threshold-based NIOM algorithm that detects changes in each metric and then clusters them to generate a continuous trace of binary occupancy.

Average Power. As Figure 1 indicates, occupancy generally implies a higher average power usage. Intuitively, the correlation between high average power and occupancy is due to occupants turning interactive loads on, which increases the home's aggregate power. To detect changes in average power $N_{\text {average }}$, at time $t$, we simply average the previous consecutive data points over a window $\tau$ in the power time-series, and then signal potential occupancy at time $t$ if the result exceeds a predefined threshold $P_{\text {average. }}$. Both $\tau$ and $P_{\text {average }}$ must be jointly set. As $\tau$ becomes smaller, to prevent false positives, $P_{\text {average }}$ should increase such that it is always greater than the maximum power over $\tau$ data points when the home is unoccupied. Similarly, as $\tau$ becomes larger, $P_{\text {average }}$ should decrease to prevent false negatives.

Standard Deviation. Figure 1 also suggests that an occupied home has more variable power usage than an unoccupied one. Intuitively, the correlation between more variable power usage and occupancy is due to occupants toggling interactive loads on and off. To detect changes in standard deviation $N_{\text {stddev }}$, at time $t$, we also compute the standard deviation of the previous $\tau$ consecutive data points in the power time-series, and then signal potential occupancy at time $t$ if the result exceeds a predefined threshold $P_{\text {stddev. As above, }}$ both $\tau$ and $P_{\text {stddev }}$ must be jointly set, since a larger $\tau$ determines $P_{\text {stddev }}$ 's magnitude. Detecting changes in standard deviation in addition to average power is useful, since occupants may toggle loads on and off frequently, such that average power does not rise above the $P_{\text {average }}$ threshold, but the standard deviation does rise above the $P_{\text {stddev }}$ threshold.

Power Range. Finally, Figure 1 suggests that occupancy implies a larger absolute range in power. We define the power range $N_{\text {range }}$ as the difference between the absolute minimum and absolute maximum power over the previous $\tau$ consecutive data points. Intuitively, the correlation between a large power range and occupancy stems from our observation that occupants sometimes have few interactions with electrical loads. While minimal interactions with electrical loads may not increase the average power or standard deviation, they are likely to increase the power's absolute range, since any interaction will briefly raise the maximum power. As above, we signal potential occupancy if the power range over the last $\tau$ data points is above a threshold $P_{\text {range }}$.

Note that we only intend our threshold-based approach above to detect daytime occupancy, e.g., 6 am to $11 \mathrm{pm}$. Nighttime occupancy correlates less with electricity usage, since occupants are generally sleeping. As a result, nighttime occupancy is much harder to determine. One simple approach, we which use in this work, is to infer nighttime occupancy from daytime occupancy: if anyone is at home at during the previous evening then we assume the home is occupied throughout the night. This works well because people are usually home at night with high probability, unless they are out of town. However, the approach may not be appropriate in some cases, e.g., if all occupants work night shifts.

Changes in each of the metrics above result in a series of discrete time-stamped occupancy events. To generate a continuous occupancy trace, we cluster these events, such that if any two events are within $\tau_{\text {cluster }}$ time window, we label the intervening period as being occupied. As $\tau_{\text {cluster }}$ increases, the greater the likelihood of false positives, e.g., detecting someone is home when no one is home, while, as $\tau_{\text {cluster }}$ decreases, the greater the likelihood of false negatives, e.g., detecting no one is home when someone is home. After clustering, occupancy detection using each metric produces a continuous time-series of the home's binary occupancy. We then combine these time-series to generate a final solution by detecting occupancy if any of the metrics signal occupancy.

Of course, for each metric, accuracy is sensitive to $\tau$ and the metric's $P$ threshold. If the power usage of the background loads is consistent, a static threshold works well. However, we investigated setting static thresholds based on training data and found that these thresholds did not work well over long periods, since the set of active background loads and their operation changed over time, e.g., as occupants turn them on and off and environmental conditions change. As a result, we set each metric's threshold dynamically based on its maximum value the previous night. Our premise is that a home's nighttime power usage and unoccupied power usage are dictated solely by its background loads. Thus, nighttime usage should provide a rough approximation of background load usage, and if any metric's value exceeds its maximum value using background loads, then the home is likely occupied.

One benefit of our approach is that it requires no training data that specifies the home's ground truth occupancy and electricity usage over an extended period. Training data is difficult to gather, since it requires deploying sensors to collect data during a training phase, which is exactly the problem NIOM is attempting to avoid. However, one drawback with our approach is that the nighttime power usage of background loads may not be an accurate indicator of background load power usage during the day. This is especially true for loads with daily cycles, such as air conditioners, which may use significantly more energy during the day. That said, our results indicate that nighttime usage performs well in our two test homes. 

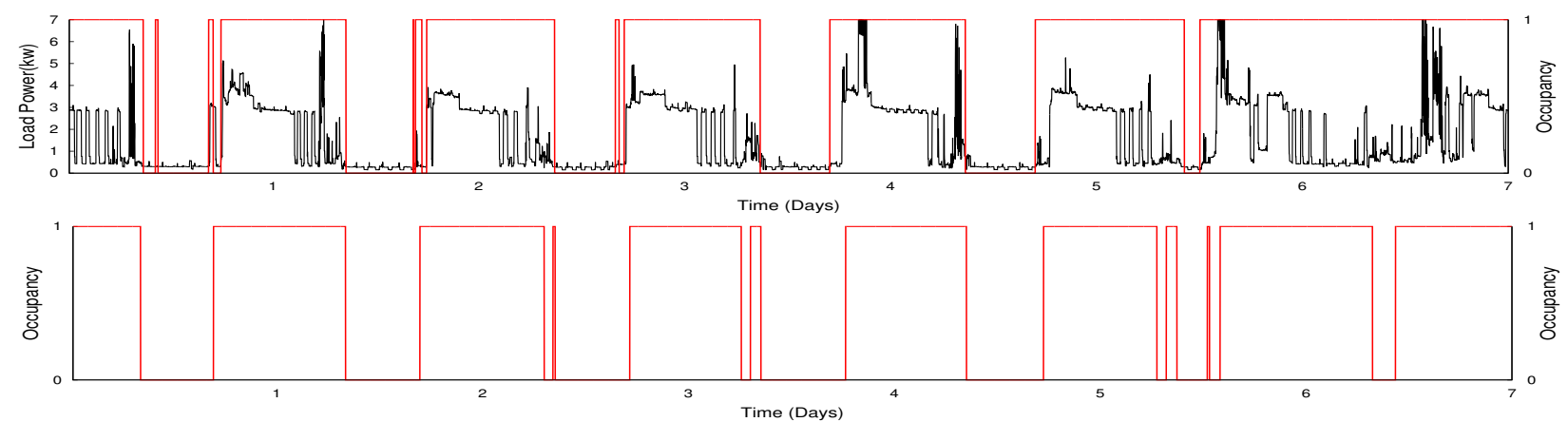

Figure 3: Home-B's load profile and ground truth occupancy over a summer week (top) and its detected occupancy using our NIOM algorithm (bottom), which combines results from all three metrics.

\begin{tabular}{|l|c|c|c|c|}
\hline & True Positives & True Negatives & False Positives & False Negatives \\
\hline Average Power & $53.50 \%$ & $24.84 \%$ & $0.54 \%$ & $21.12 \%$ \\
\hline Standard Deviation & $49.69 \%$ & $24.69 \%$ & $0.70 \%$ & $24.93 \%$ \\
\hline Power Range & $37.15 \%$ & $25.30 \%$ & $0.08 \%$ & $37.47 \%$ \\
\hline Combination & $\mathbf{6 6 . 1 1 \%}$ & $\mathbf{2 4 . 5 2 \%}$ & $\mathbf{0 . 8 6 \%}$ & $\mathbf{8 . 5 0 \%}$ \\
\hline
\end{tabular}

Table 2: NIOM accuracy for Home-B over the same summer week as Figure 3 using nighttime-based thresholds.

\section{EVALUATION}

The previous section outlines a simple threshold-based NIOM algorithm. In this section, we quantify its accuracy in two homes to demonstrate the algorithm's performance. We set $\tau=15$ data points for each metric and $\tau_{\text {cluster }}=60$ for the clustering threshold, which means our approach will generate false positives if occupants frequently leave and return within an hour. Unless otherwise noted, we set thresholds for each metric based on the metric's maximum value at night (1am-4am). Below, we compare our results with both ground truth occupancy and a NILM-based approach.

\subsection{Comparison with Ground Truth}

Figure 3 plots Home-B's load profile and ground truth occupancy over a summer week (top) and its detected occupancy using our NIOM algorithm (bottom), which combines the clustered results from all three metrics. As the graph shows, the detected occupancy is nearly identical to the ground truth occupancy. Table 2 shows the full breakdown of the percentage of time each individual metric yields true positives (detects occupancy and the home is occupied), true negatives (detects no occupancy and the home is not occupied), false positives (detects occupancy but the home is not occupied), and false negatives (detects no occupancy but the home is occupied). The overall accuracy is then $(\mathrm{TP}+\mathrm{TN}) /(\mathrm{TP}+\mathrm{TN}+\mathrm{FP}+\mathrm{FN})$. As the table shows, each metric individually generates a significant number of false negatives, with average power having the highest accuracy at $78.34 \%$. Interestingly, combining metrics significantly reduces the percentage of false negatives (by more than double in all cases) while causing only a slight increase in false positives $(<0.5 \%)$, resulting in an overall accuracy of $90.63 \%$.

The increased accuracy is due to an increase in the true positive rate (or recall), i.e., the detection accuracy given that occupants are present, from 0.72 using average power to 0.87 when combining metrics. The result demonstrates that, in this case, additional metrics beyond average power provide useful information. Since all metrics and their combination have low false positive rates, they all exhibit high precision, i.e., the probability that the home is occupied when the algorithm detects occupancy, with the combined algorithm having a precision of 0.99 . Of course, since the home is occupied $74.61 \%$ of the time, an algorithm that always assumes occupants are present will have an accuracy of $74.61 \%$. As a result, the F-measure - the harmonic mean of precision and recall-is often used instead of accuracy to assess a binary classifier's overall performance, with values closer to 1 indicating better performance. The F-measure from combining the metrics is 0.93 , while the Fmeasure of the best individual metric (average power) is only 0.70 .

Figure 4 and Table 3 shows similar results over a summer week from Home-A. Notice that Home-A's profile is significantly different than Home-B's, primarily due to its use of multiple window air conditioning units instead of a single large centralized system, as well as other high-power background loads (as shown in Figure 1(a)). In addition, the occupants in Home-A are less likely to turn off their air conditioning when they leave the home than Home-B because they own pets. As a result, occupancy detection is more challenging in Home-A. While combining the metrics still yields the best accuracy (at 79.09\%) and F-measure (at 0.82 ), each individual metric is only slightly worse. Even so, Home-A's results maintain a low rate of false positives. Home-A also demonstrates how sensitive our algorithm is to each threshold. In this case, lowering the threshold to be the average of each metric over night (rather than the maximum) significantly improves the results, yielding an overall accuracy of $90.39 \%$ and an F-measure of 0.94 .

Both experiments above examine summer periods. However, we expect the set of background loads to change each season. For instance, air conditioners have a significant influence on both load profiles above. As a result, we also examine a spring week from Home-A, which shows the weaker correlation between occupancy and electricity usage. Figure 5 and Table 4 show the results, which exhibit a higher percentage of false negatives than the summer results. The increase in false negatives is due largely to the lack of air conditioning, which narrows the difference in the load profile between an occupied and unoccupied home, as indicated in Figure 5(top). The narrowed difference makes occupancy detection more sensitive to the selection of thresholds. In addition, during the summer, occupants in both homes often manually control air conditioners-turning them on when they are home and turning them off when they leave-which makes them act more like high- 

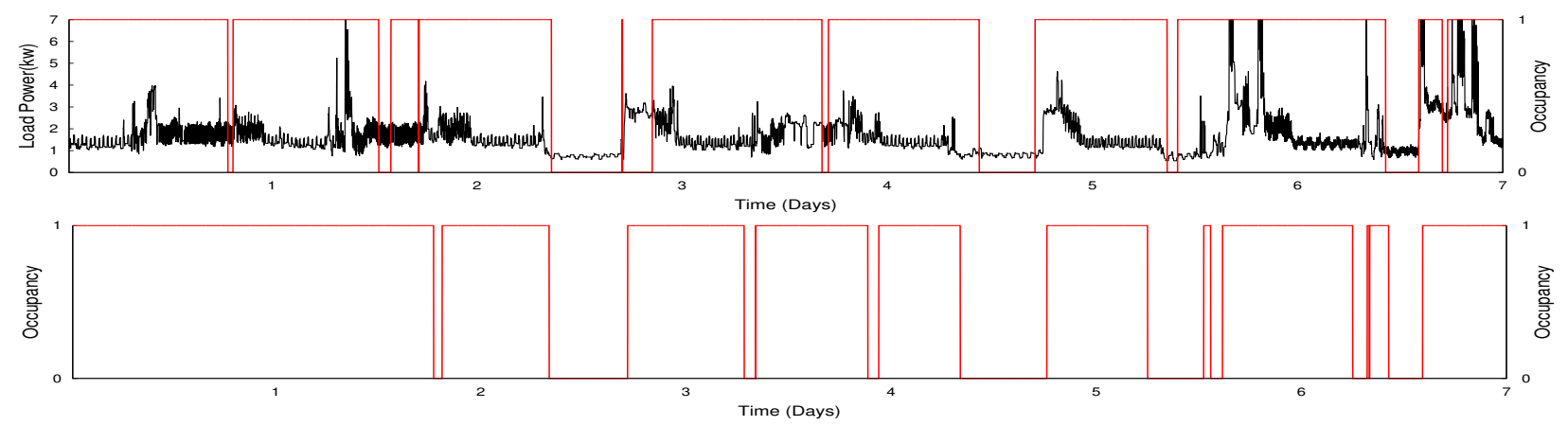

Figure 4: Home-A's load profile and ground truth occupancy over a summer week (top) and its detected occupancy using our NIOM algorithm (bottom), which combines results from all three metrics.

\begin{tabular}{|l|c|c|c|c|}
\hline & True Positives & True Negatives & False Positives & False Negatives \\
\hline Average Power & $60.82 \%$ & $11.91 \%$ & $4.39 \%$ & $22.88 \%$ \\
\hline Standard Deviation & $60.89 \%$ & $14.39 \%$ & $1.91 \%$ & $22.81 \%$ \\
\hline Power Range & $62.68 \%$ & $14.31 \%$ & $1.98 \%$ & $21.02 \%$ \\
\hline Combination & $\mathbf{6 7 . 2 7 \%}$ & $\mathbf{1 1 . 8 2 \%}$ & $\mathbf{4 . 4 7 \%}$ & $\mathbf{1 6 . 4 4 \%}$ \\
\hline
\end{tabular}

Table 3: NIOM accuracy for Home-A over the same summer week as Figure 4 using nighttime-based thresholds.

power interactive loads, rather than background loads that are consistently on regardless of occupancy. Thus, the algorithm is able to infer occupancy in the summer by detecting the presence of these loads in the aggregate data. Interestingly, in this case, the overall accuracy of $73.27 \%$ is slightly less than trivially assuming someone is always home, which yields an overall accuracy $75.3 \%$. However, the true negative rate, i.e., the accuracy given no one is home $(\mathrm{TN} /(\mathrm{TN}+\mathrm{FP}))$, is much higher $(0.86$ versus 0$)$, since the false positives remain low.

\subsection{Comparison with NILM-based Approach}

Finally, we compare the results of our simple NIOM algorithm with an approach based on a modern NILM algorithm. NILM is a well-studied problem with the ambitious goal of disaggregating a home's aggregate time-series power data to obtain the time-series power data for each of its constituent loads. One obvious way to monitor occupancy is to first perform NILM, and then analyze the resulting power usage of the interactive loads to detect occupancy. As Figure 2(b) shows, interactive loads highly correlate with occupancy, and have nearly zero power usage when occupants are not present. For comparison, we obtain the interactive load profile using a NILM algorithm, and cluster events as in $\$ 3$ to generate a continuous trace of occupancy. We then generate events in the interactive load trace from the NILM disaggregation using the same method as in $\S 2$ to detect occupants' physical interactions with loads based on the power usage of the interactive loads, e.g., any change in power $>30 \mathrm{~W}$ signals an interactive event.

We employ a NILM algorithm based on Factorial Hidden Markov Models (FHMM) used by Kolter and Johnson [22] to evaluate their Reference Energy Disaggregation Dataset (REDD), which is similar to a technique by Kim et al. [21]. FHMMs map well to NILM, since changes in building power reveal load state transitions, but not the actual state of each load. Figure 6 and Table 5 show the results. Note that our FHMM results are conservative, as we use the same data for training and disaggregation. Table 5 shows that with our standard clustering threshold of $N=60$, the NILM-based approach simply predicts $100 \%$ occupancy. This behavior is due to the fact that while NILM tends to work well for identifying background loads with regular and consistent power us- age, it has difficulty with interactive loads with no regular pattern of operation. Since interactive loads, such as lighting, do not exhibit many identifiable power features, have no regular usage patterns, and are often low-power, it is difficult for NILM algorithms to accurately extract them. As a result, as shown in Figure 6, NILM outputs large numbers of spurious events and obscures the clear periods of inactivity that signal occupancy. Lowering the clustering threshold corrects for this somewhat, as shown in Table 5, but barely improves (and eventually reduces) overall accuracy; In other words, the algorithm essentially does no better than the trivial approach of always predicting occupancy. Our comparison is only to demonstrate that the wealth of prior research on NILM does not directly apply to NIOM, and possibly other non-intrusive analytics.

\section{CONCLUSION AND FUTURE WORK}

In this paper, we explore the potential for NIOM, which analyzes electricity data from smart meters to infer occupancy, in two homes. Our intuition is that a home, when occupied, has a higher average power, standard deviation, and absolute power range over short intervals than when unoccupied. We develop a simple thresholdbased NIOM algorithm based on this intuition and then empirically evaluate its accuracy. Our results show that the algorithm performs well, especially during the summer, although dynamically setting appropriate thresholds remains a challenge. In the future, we plan to apply more sophisticated methods for associating occupancy with power usage. For example, rather than using a binary classifier, we could assign a probability of occupancy based on recent electricity usage. In addition, machine learning techniques (using our metrics as features) may perform better than simply setting ad-hoc thresholds based on a home's nighttime power usage. However, these techniques have the drawback of requiring some form of training data, which is challenging and time-consuming to collect and our current approach avoids.

While researchers have focused heavily on the problem of NILM, NIOM represents another example of the type of simple and useful analytics possible with smart meter data. As we show in $\$ 4$, directly applying a NILM algorithm to occupancy monitoring and other analytics may not be appropriate. Other potential examples of non-intrusive analytics that are distinct from NILM 


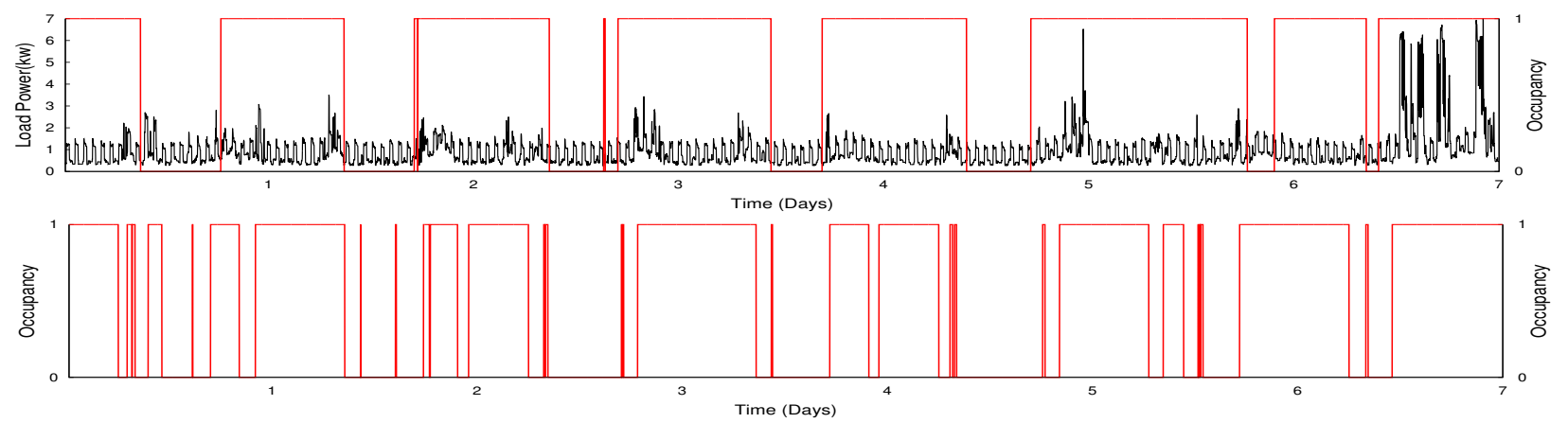

Figure 5: From top to bottom, Home-A's load profile and ground truth occupancy over a spring week, as well as occupancy detection based on the combination with thresholds for each metric set based on its maximum value at night from 1am to 4am.

\begin{tabular}{|l|c|c|c|c|}
\hline & True Positives & True Negatives & False Positives & False Negatives \\
\hline Average Power & $50.17 \%$ & $21.88 \%$ & $2.81 \%$ & $25.14 \%$ \\
\hline Standard Deviation & $38.49 \%$ & $24.08 \%$ & $0.61 \%$ & $36.82 \%$ \\
\hline Power Range & $40.64 \%$ & $23.24 \%$ & $1.45 \%$ & $34.68 \%$ \\
\hline Combination & $\mathbf{5 2 . 0 9 \%}$ & $\mathbf{2 1 . 1 8 \%}$ & $\mathbf{3 . 5 2 \%}$ & $\mathbf{2 3 . 2 1 \%}$ \\
\hline
\end{tabular}

Table 4: NIOM accuracy for Home-A over a spring week using nighttime-based thresholds.

include include separating the temperature-dependent power usage from the temperature-independent power usage, or detecting different types of loads based on common power usage characteristics. For example, in recent work, we show that resistive, inductive, and non-linear loads each present highly identifiable characteristicssmooth power growth/decay, large power spikes, and rapid and random power fluctuations-in smart meter data [5]. As another example, converting smart meter data into the frequency domain can identify cyclical loads with semi-regular duty cycles. Each of these analytics, while useful, is simpler and likely more tractable than performing a complete disaggregation using NILM.

Even with the limitations of our current approach, NIOM has compelling applications given the widespread deployment of smart meters by utilities. For instance, prior work highlights that residential users often either i) do not program or ii) mis-program programmable thermostats [24]. While the NEST thermostat attempts to address the problem by automatically programming itself based on occupancy patterns it learns using a built-in motion sensor, it carries a significant cost (roughly \$250). However, NIOM's potential to extract occupancy patterns from smart meters en masse enables utilities to automatically determine i) how much a programmable thermostat benefits each home and ii) suggest an optimal customized thermostat schedule. Utilities could include these results in electric bills or as part of routine energy audits to motivate consumers to upgrade to programmable thermostats and estimate their savings, or properly program their existing thermostat. While the applications above only require offline data, our algorithm could be run against real-time power data, although we do not evaluate its performance here. We expect future smart meters to enable customer's access to their data in real-time. Our algorithm's primary limitation in a real-time setting is its cluster threshold, which requires each metric being below its particular threshold for a certain time window before signaling occupancy. This clustering time window will dictate the system's detection latency.

Finally, our work only infers binary occupancy from smart meter data, i.e., whether anyone is home, and does not consider other dimensions of occupancy, such as the number of occupants or their location e.g.,, which room they are in. Our data suggests that the more people within a home, the more electrical events they pro- duce, although the relationship is not linear since some activities are shared. Thus, accurately monitoring a home's occupancy count would likely require a technique more advanced than our simple threshold-based approach. Monitoring occupants' location might also be possible by detecting the operation of particular loads, such as kitchen appliances, and then associating these loads with room occupancy. However, such associations would likely require some knowledge of the mapping of devices to rooms, as well as higher resolution smart meter data, since one-minute average power does not reveal many identifiable load characteristics [5].

Acknowledgements: We would like to thank both the anonymous reviewers and our shepherd, Fred Jiang, for their helpful comments and feedback, which improved the quality of this work.

\section{REFERENCES}

[1] Y. Agarwal, B. Balaji, S. Dutta, R. Gupta, and T. Weng. Duty-cycling Buildings Aggressively: The Next Frontier in HVAC Control. In IPSN, April 2011.

[2] Y. Agarwal, B. Balaji, R. Gupta, J. Lyles, M. Wei, and T. Weng. Occupancy-Driven Energy Management for Smart Building Automation. In BuildSys, November 2010.

[3] Y. Agarwal, S. Savage, and R. Gupta. SleepServer: A Software-Only Approach for Reducing the Energy Consumption of PCs within Enterprise Environments. In USENIX Annual Technical Conference, June 2010.

[4] B. Balaji, Y. Jiang, and K. Singh. A Wi-Fi Based Occupancy Sensing Approach to Smart Energy in Commercial Office Buildings. In BuildSys (Demo Session), November 2012.

[5] S. Barker, S. Kalra, D. Irwin, and P. Shenoy. Empirical Characterization and Modeling of Electrical Loads in Smart Homes. In IGCC, June 2013.

[6] S. Barker, A. Mishra, D. Irwin, E. Cecchet, P. Shenoy, and J. Albrecht. Smart*: An Open Data Set and Tools for Enabling Research in Sustainable Homes. In SustKDD, August 2012.

[7] G. Bellala, M. Marwah, M. Arlitt, G. Lyon, and C. Bash. Following the Electrons: Methods for Power Management in Commercial Buildings. In KDD, August 2012. 

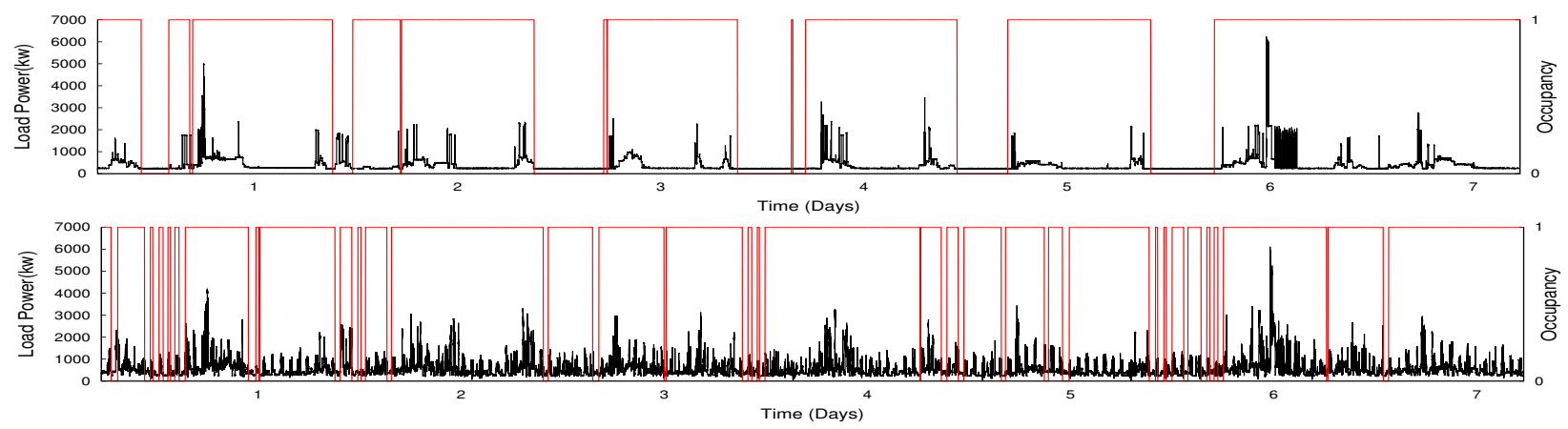

Figure 6: The power usage of the interactive loads from Home-A over the spring week (top), which highly correlate with occupancy, versus the usage of the interactive loads extracted using a NILM algorithm (bottom, $N=30$ ).

\begin{tabular}{|c|c|c|c|c|}
\hline Clustering Threshold & True Positives & True Negatives & False Positives & False Negatives \\
\hline $\mathbf{N}=\mathbf{6 0}$ & $78.61 \%$ & $0.00 \%$ & $21.39 \%$ & $0.00 \%$ \\
\hline $\mathbf{N}=\mathbf{3 0}$ & $73.30 \%$ & $6.36 \%$ & $15.03 \%$ & $5.31 \%$ \\
\hline $\mathbf{N}=\mathbf{1 5}$ & $62.58 \%$ & $11.63 \%$ & $9.76 \%$ & $16.03 \%$ \\
\hline
\end{tabular}

Table 5: Occupancy monitoring accuracy using a NILM-based approach for a week in Home-A.

[8] X. Bian, G. Abowd, and J. Regh. Using Sound Source Localization in a Home Environment. In Pervasive, May 2005.

[9] D. Delaney, G. O’Hare, and A. Ruzzelli. Evaluation of Energy-efficiency in Lighting Systems using Sensor Networks. In BuildSys, November 2009.

[10] B. Dong, B. Andrews, K. P. Lam, M. Höynck, R. Zhang, Y. Chiou, and D. Benitez. An Information Technology Enabled Sustainability Test-Bed (ITEST) for Occupancy Detection through an Environmental Sensing Network. Energy and Buildings, 42(7), July 2010.

[11] M. Ebling and M. Corner. It's all About Power and those Pesky Power Vampires. IEEE Pervasive Computing, 8(2), 2009.

[12] eGauge Energy Monitoring. http: //www. egauge. net/, 2012.

[13] U.S. Energy Information Administration, FAQ, How Many Smart Meters are Installed in the U.S. and who has them? http: //www.eia.gov/tools/faqs/faq.cfm? id $=108 \& t=3,2011$.

[14] V. Erickson and A. Cerpa. Occupancy Based Demand Response HVAC Control Strategy. In BuildSys, November 2010.

[15] V. Erickson, Y. Lin, A. Kamthe, R. Brahme, A. Cerpa, M. Sohn, and S. Narayanan. Energy Efficient Building Environment Control Strategies using Real-time Occupancy Measurements. In BuildSys, November 2009.

[16] G. Fierro, O. Rehmane, and A. Krioukov. Zone-level Occupancy Counting with Existing Infrastructure. In BuildSys (Demo sesson), November 2012.

[17] H. Gellersen, R. Want, and A. Schmidt. Bathroom Activity Monitoring Based on Sound. In Pervasive, May 2005.

[18] T. Hnat, V. Srinivasan, J. Lu, T. Sookoor, R. Dawson, J. Stankovic, and K. Whitehouse. The Hitchhiker's Guide to Successful Residential Sensing Deployments. In SenSys, November 2011.

[19] A. Kamthe, V. Erickson, M. Carreira-Perpinan, and A. Cerpa. Enabling Building Energy Auditing Using Adapted Occupancy Models. In BuildSys, November 2011.
[20] A. Kamthe, L. Jiang, M. Dudys, and A. Cerpa. SCOPES: Smart Camera Object Position Estimate System. In EWSN, February 2009.

[21] H. Kim, M. Marwah, M. Arlitt, G. Lyon, and J. Han. Unsupervised Disaggregation of Low Frequency Power Measurements. In SDM, April 2011.

[22] J. Kolter and M. Johnson. REDD: A Public Data Set for Energy Disaggregation Research. In SustKDD, August 2011.

[23] Lawrence Livermore National Laboratory, U.S. Energy Flow Chart. https://flowcharts.llnl.gov/, 2011.

[24] J. Lu, T. Sookoor, V. Srinivasan, G. Gao, B. Holben, J. Stankovic, E. Field, and K. Whitehouse. The Smart Thermostat: Using Occupancy Sensors to Save Energy in Homes. In SenSys, November 2010.

[25] C. Martani, D. Lee, P. Robinson, R. Britter, and C. Ratti. ENERNET: Studying the Dynamic Relationship between Building Occupancy and Energy Consumption. Energy and Buildings, 47, April 2012.

[26] G. Newsham and B. Birt. Building-level Occupancy Data to Improve ARIMA-based Electricity Use Forecasts. In BuildSys, 2010.

[27] S. Patel, T. Robertson, J. Kientz, M. Reynolds, and G. Abowd. At the Flick of a Switch: Detecting and Classifying Unique Electrical Events on the Residential Power Line. In Ubicomp, September 2007.

[28] A. Schoofs, D. Delaney, G. O'Hare, and A. Ruzzelli. COPOLAN: Non-Invasive Occupancy Profiling for Preliminary Assesment of HVAC Fixed Timing Strategies. In BuildSys, November 2011.

[29] E. Tapia, S. Intille, and K. Larson. Activity Recognition in the Home Setting using Simple and Ubiquitous Sensors. In Pervasive, 2004.

[30] L. Thanayankizil, S. Ghai, D. Chakraborty, and D. Seetharam. Softgreen: Towards Energy Management of Green Office Buildings with Soft Sensors. In E6 Workshop, January 2013.

[31] S. Wang and X. Jin. CO2-based Occupancy Detection for On-line Outdoor Air Flow Control. Indoor and Built Environment, 7(3), 1998. 